OPEN ACCESS

Edited by:

Junji Xing,

Houston Methodist Research Institute,

United States

Reviewed by:

Henrique Girao,

University of Coimbra, Portugal

Chaojie Wang,

The Ohio State University,

United States

*Correspondence:

Chuan Wen

chuanwen@csu.edu.cn

Jidong Tian

tianjidong@csu.edu.cn

Specialty section: This article was submitted to

Molecular Innate Immunity,

a section of the journal

Frontiers in Immunology

Received: 13 November 2020 Accepted: 31 December 2020

Published: 09 February 2021

Citation:

She Z, Xie M, Hun M, Abdirahman AS, Li C, Wu F, Luo S, Wan W, Wen C and

Tian J (2021) Immunoregulatory Effects of Mitochondria Transferred

by Extracellular Vesicles.

Front. Immunol. 11:628576.

doi: 10.3389/fimmu.2020.628576

\section{Immunoregulatory Effects of Mitochondria Transferred by Extracellular Vesicles}

\author{
Zhou She, Min Xie, Marady Hun, Amin Sheikh Abdirahman, Cuifang Li, Feifeng Wu, \\ Senlin Luo, Wuqing Wan, Chuan Wen ${ }^{*}$ and Jidong Tian*
}

Department of Pediatrics, The Second Xiangya Hospital, Central South University, Changsha, China

Mitochondria participate in immune regulation through various mechanisms, such as changes in the mitochondrial dynamics, as metabolic mediators of the tricarboxylic acid cycle, by the production of reactive oxygen species, and mitochondrial DNA damage, among others. In recent years, studies have shown that extracellular vesicles are widely involved in intercellular communication and exert important effects on immune regulation. Recently, the immunoregulatory effects of mitochondria from extracellular vesicles have gained increasing attention. In this article, we review the mechanisms by which mitochondria participate in immune regulation and exert immunoregulatory effects upon delivery by extracellular vesicles. We also focus on the influence of the immunoregulatory effects of mitochondria from extracellular vesicles to further shed light on the underlying mechanisms.

Keywords: mitochondria, extracellular vesicles, immune cell, immunoregulation, mesenchymal stem cells

\section{INTRODUCTION}

As the energy storehouse of cells, mitochondria provide ATP for cellular activities and are, thus, indispensable organelles for cell survival. Numerous studies have shown that mitochondria not only serve as energy centers of cells, but also exert important immunoregulatory effects. They participate in immune function mainly through changes in the mitochondrial dynamics. They function as metabolic mediators of the tricarboxylic acid cycle and are involved in the production of reactive oxygen species (ROS) and in mitochondrial DNA damage, among others.

Extracellular vesicles are bilayer membranous substructures produced by various cell types. They contain cellular proteins, nucleic acids, and even organelles, which can reach all parts of the body through the circulation and other body fluids, thereby constituting an important tool for intercellular communication (1). By using extracellular vesicles, mitochondria can transfer between cells and act on specific target cells, thus achieving their biological functions. This article discusses the immunoregulatory effects of mitochondria, as well as the effects of mitochondria on target cells upon delivery by extracellular vesicles. 


\section{IMMUNOREGULATORY EFFECTS OF MITOCHONDRIA}

Mitochondria are cellular energy factories of eukaryotes and the metabolic centers of cells. They serve to produce ATP through the tricarboxylic acid cycle and electron transport chain and link a variety of metabolic pathways to maintain cellular energy homeostasis. However, an increasing number of studies have shown that mitochondria can also participate in immune regulation through a variety of mechanisms (2) (Figure 1).

\section{Changes in Mitochondrial Dynamics}

Through continuous fission and fusion, mitochondria change their size, mass, and location within cells. These processes are summarized as mitochondrial dynamics (3). By regulating mitochondrial dynamics, cells can clear mitochondria that have lost their functions, repair damaged mitochondria, change the position of mitochondria, and ensure that sufficient energy supplies are provided, so as to respond to changes in the surrounding environment in a timely manner $(4,5)$.

In the case of immune cells, mitochondrial dynamics are mainly restricted to changes in the shape of the cristae to divert cellular functions. Fused mitochondria have larger cristae areas, with the cristae being closely linked, ensuring the patency of the electron transport chain, while at the same time mediating more efficient oxidative phosphorylation; on the contrary, cells with split mitochondria tend to utilize glycolysis for energy production (2). A previous study reports that naive $\mathrm{T}$ cells contain dissociated, circular mitochondria, and that their mitochondrial morphology changes through mitochondrial dynamics during activation, disconnecting the electron transport chain, increasing the production of ROS, and benefiting their own activation (6). Effector $\mathrm{T}$ cells tend to divide their mitochondria to form punctate structures, which leads to the dispersion of mitochondrial cristae, thus reducing the efficiency of the electron transport chain and promoting glycolysis. Mitochondrial fusion in memory T cells tends to form long column-shaped mitochondria, thereby enhancing the connection of the electron transport chain and improving the efficiency of oxidative phosphorylation and fatty acid oxidation. The mitochondrial intimal fusion protein, OPA1, is essential for the formation of memory $\mathrm{T}$ cells (7), which may help maintain their long-term survival. In addition, studies have shown that the fusion and fission of mitochondria may inhibit or promote the expression of interleukin (IL)-12 upon stimulation of toll-like receptors, which in turn induces $\mathrm{T}$ cells to secrete gamma interferon, thus promoting the polarization of $\mathrm{T}$ cells into Th1 cells $(8,9)$.

Mitochondrial dynamics can also affect the function of immune cells by changing the position of the mitochondria. In the process of $\mathrm{T}$ cell activation, mitochondria are dynamic and cooperate with kinesin to realize their movement to

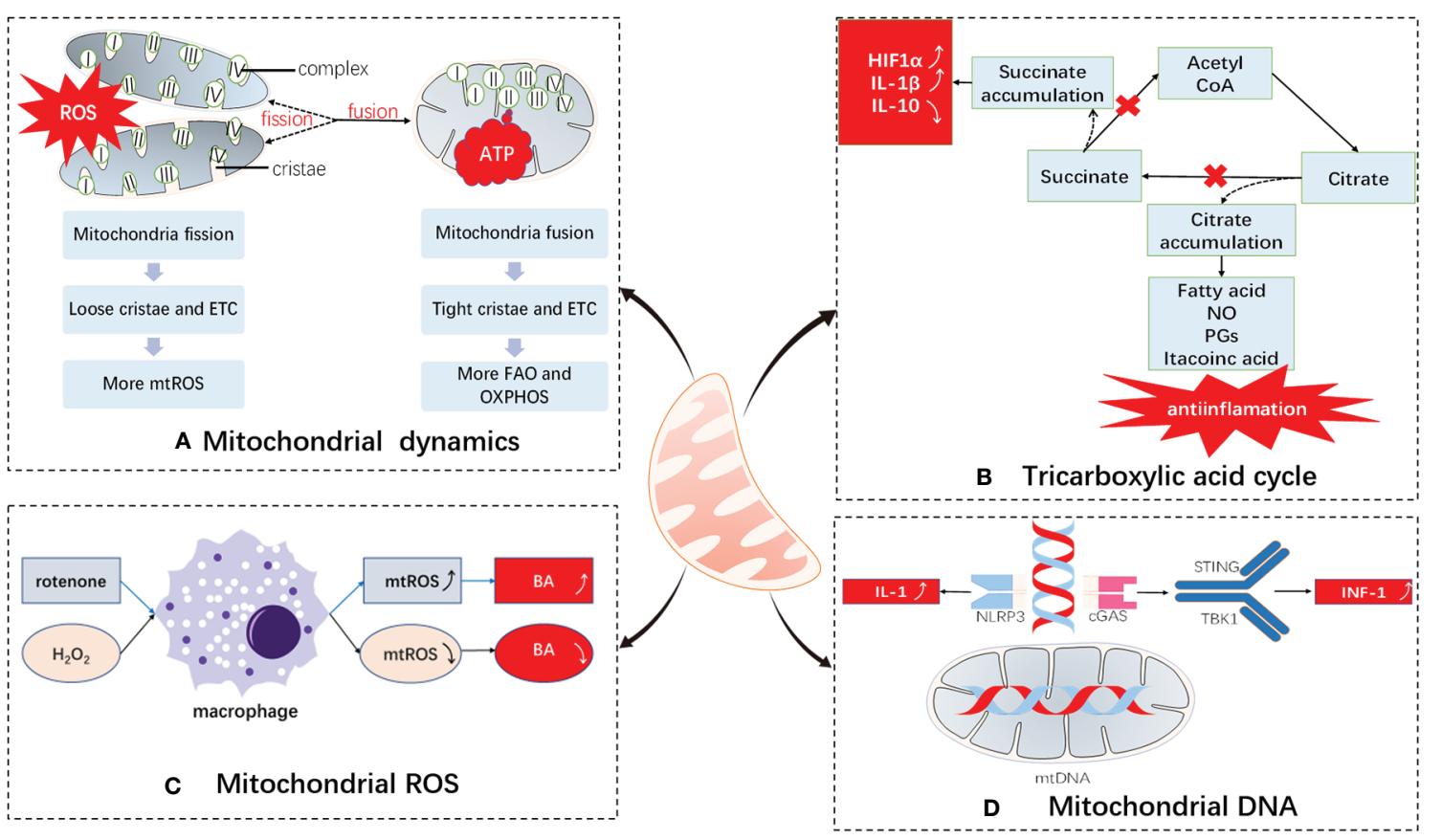

FIGURE 1 | Immunomodulatory effects of mitochondria. Mitochondria undergo (A) dynamic changes: The electron transport chains of split and fused mitochondria are different and tend to produce ROS and ATP, respectively; (B) metabolic medium of the tricarboxylic acid cycle: blockade of the tricarboxylic acid cycle leads to accumulation of inflammatory substances; (C) ROS production: mitochondrial ROS are important bactericidal substances of macrophages; (D) DNA damage participates in immune regulation: mtDNAs participate in immune responses by serving as DAMPs. ETC, electron transport chain; BA, bactericidal; ROS, reactive oxygen species; FAO, fatty acid oxidation; OXPHOS, oxidative phosphorylation; NLRP3, NACHT, LRR, and PYD domain-containing protein 3; DAMPS, damageassociated molecular patterns; cGAS, cyclic GMP-AMP synthase; STING, stimulator of interferon genes; TBK1, TANK-binding kinase 1. 
immunological synapses, to improve ATP supply and control the concentration of calcium ions to help $\mathrm{T}$ cells maintain an activated state $(10,11)$.

\section{Metabolic Mediators of the Tricarboxylic Acid Cycle}

Through the tricarboxylic acid cycle and electron transport chain, mitochondria can complete oxidative phosphorylation, thus representing the energy utilization mode of most cells (12). In addition to energy production, some metabolites of the tricarboxylic acid cycle also serve as important mediators for regulating immune responses (13).

Previous studies have shown that there are two break points in the tricarboxylic acid cycle of M1 macrophages. One occurs in the step involving isocitrate dehydrogenase (IDH), which leads to an increase in citric acid, and the other occurs in an enzymatically catalyzed step involving succinate dehydrogenase, which leads to an increase in succinate production. On the one hand, citric acid is used to synthesize fatty acids, nitric oxide, prostaglandins, and itaconic acids. The former three are key substances required for the synthesis of anti-inflammatory factors by M1 macrophages. Itaconic acid can inhibit isocitrate lyase, which prevents the growth of bacteria, including Mycobacterium tuberculosis, and also plays an antiinflammatory role $(2,6,12,14-16)$. On the other hand, succinic acid is an important pro-inflammatory factor. It can combine with hypoxia-inducible factor- $1 \alpha$ to maintain its stability to promote the production of the inflammatory mediator, IL-1 $\beta$. At the same time, it can also inhibit the production of the anti-inflammatory factor, IL-10 (12, 17-19). However, M2 macrophages are characterized by a fully functional tricarboxylic acid cycle, and do not produce too many inflammatory substances, which is in accordance with the anti-inflammatory effect of the M2 macrophages. In addition, researchers have reported that the tricarboxylic acid cycle can increase the production of interferons by increasing acetyl coenzyme A levels in the cytoplasm, which enhances the function of Th1 ancillary cells (20).

\section{Mitochondrial ROS Production}

ROS are important defense molecules of innate immunity that are generated by mitochondria and NADPH oxidase. In the transfer process of a single electron, superoxide is formed as it collides with an oxygen molecule $(\mathrm{O} 2)$ at a specific location (such as complexes I and III), resulting in $\operatorname{ROS}(21,22)$. Initially, they have been proposed to kill bacteria directly by chemical action and were considered a by-product of monocyte respiration (23, 24). Further research has shown that mitochondrial ROS (mtROS) have important effects on immune regulation (22). Uncoupling protein 2 (UCP2) is a negative regulator of mtROS. UCP2-deficient mice exhibit stronger resistance to Toxoplasma gondii and Salmonella typhimurium infections, as well as macrophages with stronger cytokine-release potential $(22,25)$. Previously, researchers have also used rotenone to inhibit the electron transport chain of macrophages, resulting in a significant increase in mtROS. It has been reported that the production of IL-1 $\beta$ by macrophages is significantly increased in a dose-dependent manner (26). On the contrary, when activated macrophages are exposed to hydrogen peroxide, their antibacterial activity decreases significantly (27).

For adaptive immune cells, mtROS are also important mediators of associated immune functions. It was found that mtROS are essential for activating $\mathrm{T}$ cell nuclear factors and $\mathrm{T}$ cells to produce IL-2 (28). Moreover, after inhibiting glycerol-3phosphate dehydrogenase 2 (GPD2, an enzyme that produces mtROS) in T cells, IL-2 production is reduced $(2,29)$. Early B cells undergo somatic hypermutation and class-switch recombination to change the properties of the antigen receptor and immune globulin molecules, and eventually differentiate into memory B cells or plasma cells. Previous studies have shown that increasing mitochondrial mass and mtROS of B cells can inhibit heme synthesis, relieve the antagonistic effect of BACH2 (antigen category conversion for necessary transcription factors), and enhance class-switch recombination $(2,6)$.

\section{Mitochondrial DNA Damage}

Mitochondrial DNA is mainly involved in immunity as a damage-related model molecule, more specifically, a damageassociated molecular pattern (DAMP) (30). During apoptosis, mitochondria release oxidized mitochondrial DNA and bind to NLRP3 inflammatory bodies, thus promoting the activation of downstream cell apoptotic protease- 1 and the production of IL-1 $(2,31)$. Thus, mitochondrial DNA establishes a link between mitochondrial damage and apoptotic protease-1 (32).

Another substance binding to the mitochondrial DNA is cyclic guanosine monophosphate-adenosine monophosphate (cGAMP) synthase (cGAS) $(33,34)$. When mitochondrial DNA enters the cytoplasm, it combines with cGAS to promote the formation of cGAMP, which serves as a second messenger, and acts in conjunction with downstream mediators, such as stimulator of interferon genes (STING) and TANK binding kinase-1 (TBK1), and promotes the formation of type 1 interferons, thus playing an important anti-viral role (35-37).

\section{IMMUNOMODULATORY EFFECTS OF EXTRACELLULAR VESICLES}

Extracellular vesicles are a type of bilayer membrane vesicles released by cells, with diameters ranging from 40 to $2000 \mathrm{~nm}$. They contain a variety of substances, including DNA, proteins, lipids, and organelles, among others, which can be exchanged between cells with the help of the internal environment $(1,38$ 41). Material exchange through extracellular vesicles provides the following advantages: 1. Protection: The membrane structure of extracellular vesicles can protect the contents from degradation; 2. Targeting: The unique markers on the surface of extracellular vesicles can bind to the corresponding receptors on the target cells to achieve specific targeting; 3. Enrichment: The contents of the extracellular vesicles are released around or inside the target cells, which can locally increase the concentration of the substances released (42). Owing to their 
unique contents and the ability to act on intercellular communication, extracellular vesicles can be used as disease markers, therapeutic targets, and drug carriers, and have great potential in the diagnosis, monitoring, and treatment of diseases (1, 39, 41, 43-46). For example, in cardiovascular diseases, extracellular vesicles may promote the development and progression of atherosclerosis by promoting initial lesion formation, intravascular calcification, unstable plaque progression and post-rupture thrombosis, while extracellular vesicles released from stem cells have the function of promoting capillary angiogenesis, reducing infarct size, and oxidative stress (47); in metabolic diseases, extracellular vesicles may regulate insulin resistance, or macrophage phenotype, thus affecting disease progression $(48,49)$.

Extracellular vesicles play an important role in immunity by transferring protein components, such as cytokines, major histocompatibility complexes (MHC), and annexin (50). Dendritic cells, macrophages, and mast cells release extracellular vesicles containing MHC-II molecules, which serve to transfer them to target cells to influence antigen presentation and activate cellular immunity (50-52). It has been shown that macrophages infected with Mycobacterium tuberculosis can release MHC-II and Mycobacterium tuberculosis antigen, thus activating $\mathrm{T}$ cells and cellular immunity $(53,54)$.

In addition to proteins, extracellular vesicles can also carry miRNAs with various immunomodulatory effects (1). In a mouse model of myocardial ischemia and reperfusion, mesenchymal stromal cells secreted exosomes loaded with miRNA-182, which were shown to target the TLR4/NF- $\kappa \mathrm{B} / \mathrm{PI} 3 \mathrm{~K} / \mathrm{Akt}$ pathway, stimulate macrophages to differentiate into the M2 type, play an anti-inflammatory role, and reduce damage (55). In addition to macrophages, exosomes from mesenchymal stem cells have been reported to carry miR-let7, which can induce M2 macrophage transformation through the HMGA2/NF- $\kappa \mathrm{B}$ pathway and ameliorate atherosclerosis (56). In a mouse model of septicemia, miR-146a is shown to target the TRAF6 pathway, consequently promoting M2 macrophage polarization and prolonging the survival time of mice (57).

\section{IMMUNOREGULATORY EFFECTS OF MITOCHONDRIA TRANSFERRED BY EXTRACELLULAR VESICLES}

Mediated by arrestin domain-containing protein 1(ARRDC1), cells can release extracellular vesicles containing mitochondria $(58,59)$, a process that may be mediated by CD-38 $(60)$, but the exact form remains to be investigated (Figure 2).

Previous studies identified mitochondria to be enclosed in extracellular vesicles, based on which they were considered to be related to the pathogenesis of certain diseases and disease markers (Table 1). For example, some researchers extracted extracellular vesicles containing mitochondria from blood samples of patients with systemic lupus erythematosus, and the concentration of those vesicles was shown to be related to disease activity and the concentration of the inflammatory factors. Further research revealed that extracellular vesicles carrying mitochondria may be involved in the pathogenesis of lupus

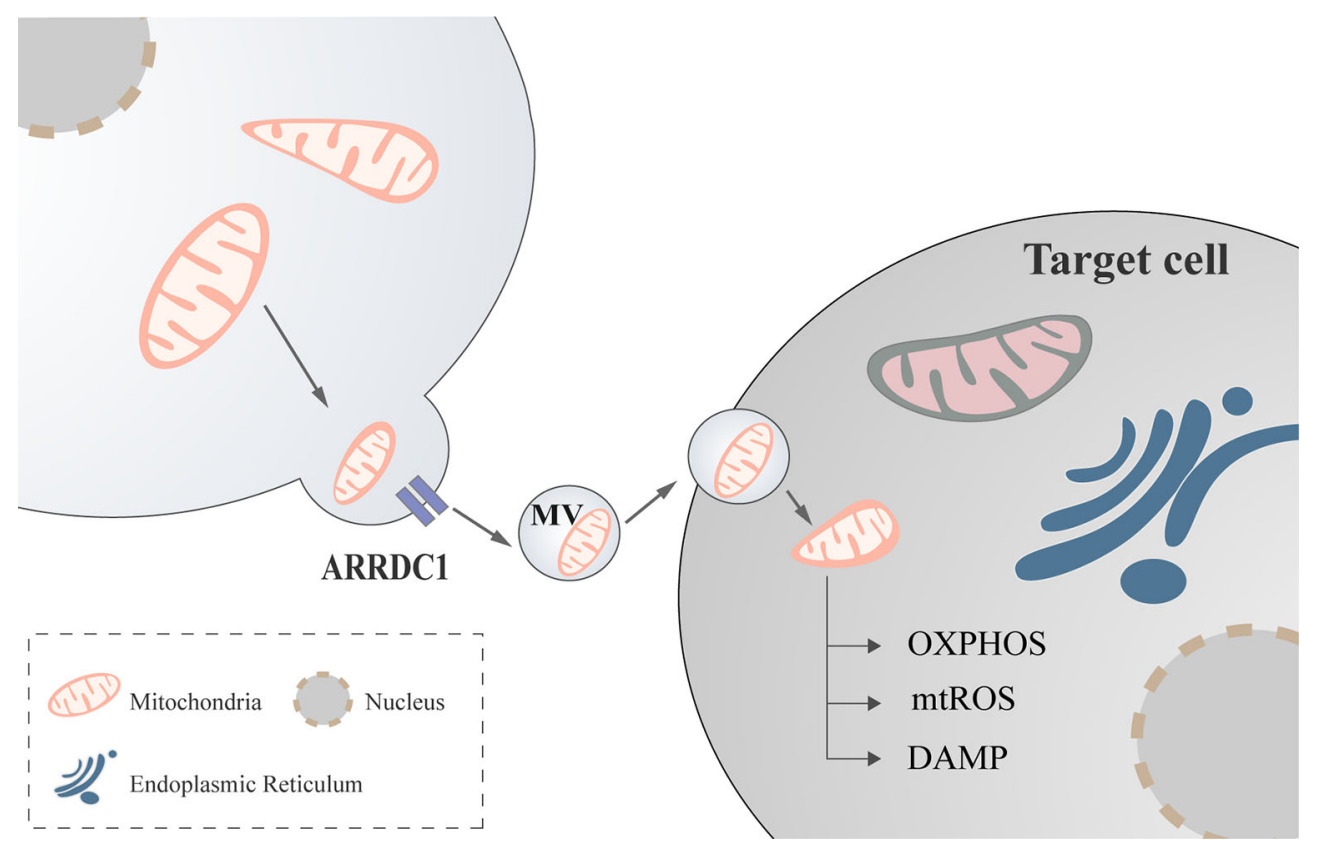

FIGURE 2 | Mitochondria regulate immune cell via extracellular vesicles. Mediated by ARRDC1, mitochondria communicate between cells with the help of extracellular vesicles to modulate cellular functions. ARRDC1, arrestin domain-containing protein 1. 
TABLE 1 | Mitochondrial immunomodulatory effects upon delivery by extracellular vesicles.

\begin{tabular}{|c|c|c|c|c|}
\hline Origin of EVs & Target cells & Direct effects & Indirect effects & Reference \\
\hline Patient melanoma metastatic tissues & - & Biomarker of tumor cells & - & $(61)$ \\
\hline Blood samples of patients with SLE & - & Promote inflammation & Related to disease activity & (62) \\
\hline BM-MSCs & Alveolar epithelium & Increase in ATP levels & Protect against ALI & (63) \\
\hline hBM-MSCs & Macrophage & Increase in ATP and ROS levels & React to Oxidative stress & (64) \\
\hline MSCs & Macrophage & Enhance bioenergetics & Regulate macrophage functions & (58) \\
\hline Astrocytes & - & Increase in ATP levels & - & (65) \\
\hline Astrocytes & Neurons & Increase in ATP levels & Promote survival and plasticity & (60) \\
\hline MDRCs & T cells & Co-localize with the mitochondrial network of $T$ cells & Increase ROS generation & (66) \\
\hline LPS-stimulated monocytic cells & Endothelial cells & Mediators of communication & Promote inflammation & (67) \\
\hline Platelets & Neutrophils & Substrate for the bactericidal secreted phospholipase A2-IIA & Regulate immune reactions & (68) \\
\hline
\end{tabular}

BM-MSCs, Bone marrow-derived stromal cells; hBM-MSCs, human bone marrow-derived stromal cells; MSCs, mesenchymal stem cells; MDRCs, airway myeloid-derived regulatory cells, ALI, acute lung injury.

erythematosus (62). Similarly, some researchers extracted extracellular vesicles from the plasma of patients with melanoma, which contained mitochondrial proteins. The latter may become unique marker proteins of extracellular vesicles associated with melanoma, as they are different from canonical extracellular vesicles (61).

As mentioned above, extracellular vesicles themselves constitute an important tool for intercellular communication, along with immunomodulatory effects $(1,40,69)$. In addition, mitochondria can participate in immune regulation in various ways and exert direct or indirect regulatory effects on immune cells. For example, they affect the selective differentiation of B cells and the production of antibodies (70). When T cells are activated, their mitochondria assemble into specialized structures to produce sufficient amounts of one-carbon units (71). Therefore, it is worth exploring whether mitochondria play a role in immune regulation through extracellular vesicles.

Transferred by extracellular vesicles, mitochondria can regulate the function of other cells, such as alveolar epithelial cells, macrophages, nerve cells (neurons), neutrophils, and endothelial cells, and complete the communication between cells. Most transferred mitochondria achieve their functions in terms of regulation, protection, and recovery by increasing oxidative phosphorylation/energy production in target cells. For example, bone marrow-derived mesenchymal stem cells can transfer mitochondria to alveolar epithelial cells through extracellular vesicles, consequently improving energy production by epithelial and surrounding cells, restoring the function of type II alveolar epithelial cells to produce alveolar active substances, thus protecting alveolar epithelial cells in the case of acute lung injury, and improving the survival rate of mice with acute lung injury stimulated by lipopolysaccharide (63). However, how do transferred mitochondria protect the lungs by regulating immunity? Using a model of acute respiratory distress, researchers have found that mesenchymal stem cells could use extracellular vesicles to transfer mitochondria to alveolar macrophages. This led to an improvement in the oxidative phosphorylation ability of alveolar macrophages, and to their differentiation into the anti-inflammatory M2 type. It further served to enhance the phagocytic capacity of M2-type alveolar macrophages and reduce the secretion of TNF- $\alpha$, thus reducing lung inflammation (64). In addition, studies have found that extracellular vesicles derived from mesenchymal stem cells could transfer mitochondria to macrophages and fuse with the mitochondrial network of macrophages, thus increasing ATP production (58).

The transported mitochondria are required to be functional $(60,63,64)$. After a stroke, astrocytes use extracellular vesicles to transfer mitochondria to neurons through a CD38-dependent mechanism, which can protect cells from hypoxia and glucose deprivation, and improve the plasticity of cells after injury. It is worth exploring whether CD38 is also expressed in immune cells. In this series of experiments, no evidence was found that inhibition of CD38 could affect the release of mitochondria from immune cells; therefore, further research and discussion are warranted (60).

Moreover, platelets have been described to secrete vesicular mitochondria, which are decomposed by phospholipase A2-IIA (sPLA2-IIA) after being absorbed by neutrophils, followed by the release of mitochondrial DNA, lysophospholipids, and fatty acids. These substances can be used as damage-related model molecules to stimulate neutrophil activation and promote their pro-inflammatory responses (68). By serving as an energy source, the amount of energy provided by mitochondria is also a means of regulating an immune response. As mentioned above, in a disease model of acute respiratory distress syndrome, after the mitochondria derived from mesenchymal stem cells had entered the alveolar macrophages, they could enhance the oxidative phosphorylation capacity and promote the antiinflammatory expression profile and phagocytic functions of macrophages, and reduce lung inflammation (64). Moreover, the mitochondrial network that is integrated into $\mathrm{T}$ cells was shown to affect the mitotic processes of $\mathrm{T}$ cells, highlighting another means by which extracellular vesicle mitochondria exert their effects (66).

In order to study the effect of activated monocyte vesicles on endothelial cell inflammation, Puhm et al. stimulated monocytes with lipopolysaccharide to produce extracellular vesicles containing mitochondria. These vesicles could induce type I interferon and tumor necrosis factor responses in endothelial cells. However, clearing these mitochondria or directly inhibiting the function of mitochondria in monocytes (pyruvate inhibition or use of a reactive oxygen scavenger to reduce ROS) could reduce the inflammatory effect of these vesicles on endothelial 
cells (67). This experiment highlighted that mitochondria in extracellular vesicles can stimulate the production of inflammatory responses and influence immune regulation.

\section{FUTURE PERSPECTIVES}

In conclusion, mitochondria not only supply energy to the cells, but also participate in immune regulation, directly or indirectly, through a variety of mechanisms. They are important immuneregulatory centers that have been widely studied. Moreover, mitochondrial dysfunction is also associated with many autoimmune diseases (72-74). The immune regulation of extracellular vesicles is a novel and important research hotspot. With the help of extracellular vesicles, mitochondria allow for communication between cells and better participation in the regulation of the immune system. These findings provide a direction for future extracellular vesicle-based or mitochondrial precision therapies. Although the ability of mitochondria to regulate immune cells through extracellular vesicles has been

\section{REFERENCES}

1. Xie M, Xiong W, She Z, Wen Z, Abdirahman AS, Wan W, et al. Immunoregulatory Effects of Stem Cell-Derived Extracellular Vesicles on Immune Cells. Front Immunol (2020) 11:13. doi: 10.3389/fimmu.2020.00013

2. Angajala A, Lim S, Phillips JB, Kim JH, Yates C, You Z, et al. Diverse Roles of Mitochondria in Immune Responses: Novel Insights Into ImmunoMetabolism. Front Immunol (2018) 9:1605. doi: 10.3389/fimmu.2018.01605

3. Liesa M, Shirihai OS. Mitochondrial Networking in T Cell Memory. CELL (2016) 166:9-10. doi: 10.1016/j.cell.2016.06.035

4. Horbay R, Bilyy R. Mitochondrial dynamics during cell cycling. APOPTOSIS (2016) 21:1327-35. doi: 10.1007/s10495-016-1295-5

5. Lisowski P, Kannan P, Mlody B, Prigione A. Mitochondria and the dynamic control of stem cell homeostasis. EMBO Rep (2018) 19(5):e45432. doi: 10.15252/embr.201745432

6. Mills EL, Kelly B, O’Neill LAJ. Mitochondria are the powerhouses of immunity. Nat Immunol (2017) 18:488-98. doi: 10.1038/ni.3704

7. Buck MD, Sullivan D O, Klein Geltink RI, Curtis JD, Chang C, Sanin DE, et al. Mitochondrial Dynamics Controls T Cell Fate through Metabolic Programming. Cell (2016) 166:63-76. doi: 10.1016/j.cell.2016.05.035

8. Vignali DAA, Kuchroo VK. IL-12 family cytokines: immunological playmakers. Nat Immunol (2012) 13:722-8. doi: 10.1038/ni.2366

9. Gao Z, Li Y, Wang F, Huang T, Fan K, Zhang Y, et al. Mitochondrial dynamics controls anti-tumour innate immunity by regulating CHIP-IRF1 axis stability. Nat Commun (2017) 8(1):1805. doi: 10.1038/s41467-01701919-0

10. Quintana A, Hoth M. Mitochondrial dynamics and their impact on T cell function. Cell Calcium (2012) 52:57-63. doi: 10.1016/j.ceca.2012.02.005

11. Desdín-Micó G, Soto-Heredero G, Mittelbrunn M. Mitochondrial activity in T cells. Mitochondrion (2018) 41:51-7. doi: 10.1016/j.mito.2017.10.006

12. O’Neill LAJ, Kishton RJ, Rathmell J. A guide to immunometabolism for immunologists. Nat Rev Immunol (2016) 16:553-65. doi: 10.1038/nri.2016.70

13. Sander LE, Garaude J. The mitochondrial respiratory chain: A metabolic rheostat of innate immune cell-mediated antibacterial responses. Mitochondrion (2018) 41:28-36. doi: 10.1016/j.mito.2017.10.008

14. Shapiro H, Lutaty A, Ariel A. Macrophages, Meta-Inflammation, and Immuno-Metabolism. Sci World J (2011) 11:2509-29. doi: 10.1100/2011/ 397971

15. Michelucci A, Cordes T, Ghelfi J, Pailot A, Reiling N, Goldmann O, et al. Immune-responsive gene 1 protein links metabolism to immunity by highlighted, there are still many unknown mechanisms that need to be addressed in future studies.

\section{AUTHOR CONTRIBUTIONS}

ZS and MX prepared the table and figure. ZS, MH, AA, CL, FW, SL, WW, JT, and CW drafted the manuscript. ZS and CW edited and revised the manuscript. All authors contributed to the article and approved the submitted version.

\section{FUNDING}

This work was supported by grants from the Natural Science Foundation of Hunan Province in China (grant no. 2019JJ40413), the National Natural Science Foundation of China (grant no. 82070758), and Hunan Provincial Key R\&D Program Project (grant no. 2020SK2084). catalyzing itaconic acid production. Proc Natl Acad Sci (2013) 110:7820-5. doi: $10.1073 /$ pnas. 1218599110

16. Jha AK, Huang SC, Sergushichev A, Lampropoulou V, Ivanova Y, Loginicheva E, et al. Network Integration of Parallel Metabolic and Transcriptional Data Reveals Metabolic Modules that Regulate Macrophage Polarization. Immunity (2015) 42:419-30. doi: 10.1016/j.immuni.2015.02.005

17. Tannahill GM, Curtis AM, Adamik J, Palsson-McDermott EM, McGettrick AF, Goel G, et al. Succinate is an inflammatory signal that induces IL-1 $\beta$ through HIF-1 $\alpha$. Nature (2013) 496:238-42. doi: 10.1038/nature11986

18. Mills E, O Neill LAJ. Succinate: a metabolic signal in inflammation. Trends Cell Biol (2014) 24:313-20. doi: 10.1016/j.tcb.2013.11.008

19. Mills EL, Kelly B, Logan A, Costa ASH, Varma M, Bryant CE, et al. Succinate Dehydrogenase Supports Metabolic Repurposing of Mitochondria to Drive Inflammatory Macrophages. Cell (2016) 167:457-70. doi: 10.1016/ j.cell.2016.08.064

20. Bailis W, Shyer JA, Zhao J, Canaveras JCG, Al Khazal FJ, Qu R, et al. Distinct modes of mitochondrial metabolism uncouple $\mathrm{T}$ cell differentiation and function. Nature (2019) 571:403-7. doi: 10.1038/s41586-019-1311-3

21. Brand MD. The sites and topology of mitochondrial superoxide production. Exp Gerontol (2010) 45:466-72. doi: 10.1016/j.exger.2010.01.003

22. Sena LA, Chandel NS. Physiological Roles of Mitochondrial Reactive Oxygen Species. Mol Cell (2012) 48:158-67. doi: 10.1016/j.molcel.2012.09.025

23. Rada B, Leto TL. Oxidative innate immune defenses by Nox/Duox family NADPH oxidases. Contributions Microbiol (2008) 15:164. doi: 10.1159/ 000136357

24. Dan Dunn J, Alvarez LA, Zhang X, Soldati T. Reactive oxygen species and mitochondria: A nexus of cellular homeostasis. Redox Biol (2015) 6:472-85. doi: 10.1016/j.redox.2015.09.005

25. Rousset S, Emre Y, Joinlambert O, Hurtaud C, Ricquier D, Cassarddoulcier A. The uncoupling protein 2 modulates the cytokine balance in innate immunity ir. Cytokine (2006) 35:135-42. doi: 10.1016/j.cyto.2006.07.012

26. Zhou R, Yazdi AS, Menu P, Tschopp J. A role for mitochondria in NLRP3 inflammasome activation. Nature (2011) 469:221-5. doi: 10.1038/nature09663

27. West AP, Brodsky IE, Rahner C, Woo DK, Erdjument-Bromage H, Tempst P, et al. TLR signalling augments macrophage bactericidal activity through mitochondrial ROS. Nature (2011) 472:476-80. doi: 10.1038/nature09973

28. Sena LA, Li S, Jairaman A, Prakriya M, Ezponda T, Hildeman DA, et al. Mitochondria Are Required for Antigen-Specific T Cell Activation through Reactive Oxygen Species Signaling. Immunity (2013) 38:225-36. doi: 10.1016/ j.immuni.2012.10.020 
29. Kamiński MM, Sauer SW, Kamiński M, Opp S, Ruppert T, Grigaravičius P, et al. T cell Activation Is Driven by an ADP-Dependent Glucokinase Linking Enhanced Glycolysis with Mitochondrial Reactive Oxygen Species Generation. Cell Rep (2012) 2:1300-15. doi: 10.1016/j.celrep.2012.10.009

30. Banoth B, Cassel SL. Mitochondria in innate immune signaling. Transl Res (2018) 202:52-68. doi: 10.1016/j.trsl.2018.07.014

31. Shimada K, Crother TR, Karlin J, Dagvadorj J, Chiba N, Chen S, et al. Oxidized Mitochondrial DNA Activates the NLRP3 Inflammasome during Apoptosis. Immunity (2012) 36:401-14. doi: 10.1016/j.immuni.2012.01.009

32. Nakahira K, Haspel JA, Rathinam VAK, Lee S, Dolinay T, Lam HC, et al. Autophagy proteins regulate innate immune responses by inhibiting the release of mitochondrial DNA mediated by the NALP3 inflammasome. Nat Immunol (2011) 12:222-30. doi: 10.1038/ni.1980

33. West AP, Khoury-Hanold W, Staron M, Tal MC, Pineda CM, Lang SM, et al. Mitochondrial DNA stress primes the antiviral innate immune response. Nature (2015) 520:553-7. doi: 10.1038/nature14156

34. Moriyama $\mathrm{M}$, Koshiba $\mathrm{T}$, Ichinohe $\mathrm{T}$. Influenza A virus $\mathrm{M} 2$ protein triggers mitochondrial DNA-mediated antiviral immune responses. Nat Commun (2019) 10(1):4624. doi: 10.1038/s41467-019-12632-5

35. Lood C, Blanco LP, Purmalek MM, Carmona-Rivera C, De Ravin SS, Smith CK, et al. Neutrophil extracellular traps enriched in oxidized mitochondrial DNA are interferogenic and contribute to lupus-like disease. Nat Med (2016) 22:146-53. doi: $10.1038 / \mathrm{nm} .4027$

36. Majid A. Mapping words reveals emotional diversity. Science (2019) 366:1444-5. doi: 10.1126/science.aba1763

37. Riley JS, Tait SW. Mitochondrial DNA in inflammation and immunity. EMBO Rep (2020) 21:e49799. doi: 10.15252/embr.201949799

38. Colombo M, Raposo G, Thery C. Biogenesis, secretion, and intercellular interactions of exosomes and other extracellular vesicles. Annu Rev Cell Dev Biol (2014) 30:255-89. doi: 10.1146/annurev-cellbio-101512-122326

39. Shao H, Im H, Castro CM, Breakefield X, Weissleder R, Lee H. New Technologies for Analysis of Extracellular Vesicles. Chem Rev (2018) 118:1917-50. doi: 10.1021/acs.chemrev.7b00534

40. Macia L, Nanan R, Hosseini-Beheshti E, Grau GE. Host- and MicrobiotaDerived Extracellular Vesicles, Immune Function, and Disease Development. Int J Mol Sci (2020) 21:107. doi: 10.3390/ijms21010107

41. Sun X, Meng H, Wan W, Xie M, Wen C. Application potential of stem/ progenitor cell-derived extracellular vesicles in renal diseases. Stem Cell Res Ther (2019) 10(1):8. doi: 10.1186/s13287-018-1097-5

42. Fitzgerald W, Freeman ML, Lederman MM, Vasilieva E, Romero R, Margolis L. A System of Cytokines Encapsulated in ExtraCellular Vesicles. Sci Rep-Uk (2018) 8 (1):8973. doi: 10.1038/s41598-018-27190-x

43. Lener T, Gimona M, Aigner L, Börger V, Buzas E, Camussi G, et al. Applying extracellular vesicles based therapeutics in clinical trials - an ISEV position paper. J Extracell Vesicles (2015) 4:30087. doi: 10.3402/jev.v4.30087

44. Boere J, Malda J, van de Lest CHA, van Weeren PR, Wauben MHM. Extracellular Vesicles in Joint Disease and Therapy. Front Immunol (2018) 9:2575. doi: 10.3389/fimmu.2018.02575

45. Lan B, Zeng S, Grützmann R, Pilarsky C. The Role of Exosomes in Pancreatic Cancer. Int J Mol Sci (2019) 20:4332. doi: 10.3390/ijms20184332

46. McVey MJ, Maishan M, Blokland KEC, Bartlett N, Kuebler WM. Extracellular vesicles in lung health, disease, and therapy. Am J Physiol-Lung C (2019) 316: L977-89. doi: 10.1152/ajplung.00546.2018

47. Boulanger CM, Loyer X, Rautou P, Amabile N. Extracellular vesicles in coronary artery disease. Nat Rev Cardiol (2017) 14:259-72. doi: 10.1038/ nrcardio.2017.7

48. Pardo F, Villalobos-Labra R, Sobrevia B, Toledo F, Sobrevia L. Extracellular vesicles in obesity and diabetes mellitus. Mol Aspects Med (2018) 60:81-91. doi: 10.1016/j.mam.2017.11.010

49. Akbar N, Azzimato V, Choudhury RP, Aouadi M. Extracellular vesicles in metabolic disease. Diabetologia (2019) 62:2179-87. doi: 10.1007/s00125-01905014-5

50. Groot Kormelink T, Mol S, de Jong EC, Wauben MHM. The role of extracellular vesicles when innate meets adaptive. Semin Immunopathol (2018) 40:439-52. doi: 10.1007/s00281-018-0681-1

51. Chen Z, Larregina AT, Morelli AE. Impact of extracellular vesicles on innate immunity. Curr Opin Organ Tran (2019) 24:670-8. doi: 10.1097/ MOT.0000000000000701
52. Kowal J, Tkach M. Dendritic cell extracellular vesicles. Int Rev Cell Mol Biol (2019) 349:213-49. doi: 10.1016/bs.ircmb.2019.08.005

53. Giri PK, Schorey JS. Exosomes derived from M. Bovis BCG infected macrophages activate antigen-specific $\mathrm{CD} 4+$ and $\mathrm{CD} 8+\mathrm{T}$ cells in vitro and in vivo. PloS One (2008) 3:e2461. doi: 10.1371/journal.pone.0002461

54. Ramachandra L, Qu Y, Wang Y, Lewis CJ, Cobb BA, Takatsu K, et al. Mycobacterium tuberculosis Synergizes with ATP To Induce Release of Microvesicles and Exosomes Containing Major Histocompatibility Complex Class II Molecules Capable of Antigen Presentation. Infect Immun (2010) 78:5116-25. doi: 10.1128/IAI.01089-09

55. Zhao J, Li X, Hu J, Chen F, Qiao S, Sun X, et al. Mesenchymal stromal cellderived exosomes attenuate myocardial ischaemia-reperfusion injury through miR-182-regulated macrophage polarization. Cardiovasc Res (2019) 115:1205-16. doi: 10.1093/cvr/cvz040

56. Li J, Xue H, Li T, Chu X, Xin D, Xiong Y, et al. Exosomes derived from mesenchymal stem cells attenuate the progression of atherosclerosis in ApoE- $/$ mice via miR-let7 mediated infiltration and polarization of M2 macrophage. Biochem Bioph Res Co (2019) 510:565-72. doi: 10.1016/j.bbrc.2019.02.005

57. Song Y, Dou H, Li X, Zhao X, Li Y, Liu D, et al. Exosomal miR-146a Contributes to the Enhanced Therapeutic Efficacy of Interleukin-1 $\beta$-Primed Mesenchymal Stem Cells Against Sepsis. Stem Cells (2017) 35:1208-21. doi: $10.1002 /$ stem.2564

58. Phinney DG, Di Giuseppe M, Njah J, Sala E, Shiva S, St Croix CM, et al. Mesenchymal stem cells use extracellular vesicles to outsource mitophagy and shuttle microRNAs. Nat Commun (2015) 6:8472. doi: 10.1038/ncomms9472

59. Liu D, Dong Z, Wang J, Tao Y, Sun X, Yao X. The existence and function of mitochondrial component in extracellular vesicles. Mitochondrion (2020) 54:122-7. doi: 10.1016/j.mito.2020.08.005

60. Hayakawa K, Esposito E, Wang X, Terasaki Y, Liu Y, Xing C, et al. Transfer of mitochondria from astrocytes to neurons after stroke. Nature (2016) 535:5515. doi: $10.1038 /$ nature 18928

61. Jang SC, Crescitelli R, Cvjetkovic A, Belgrano V, Olofsson Bagge R, Sundfeldt K, et al. Mitochondrial protein enriched extracellular vesicles discovered in human melanoma tissues can be detected in patient plasma. J Extracell Vesicles (2019) 8:1635420. doi: $10.1080 / 20013078.2019 .1635420$

62. Mobarrez F, Fuzzi E, Gunnarsson I, Larsson A, Eketjäll S, Pisetsky DS, et al. Microparticles in the blood of patients with SLE: Size, content of mitochondria and role in circulating immune complexes. J Autoimmun (2019) 102:142-9. doi: 10.1016/j.jaut.2019.05.003

63. Islam MN, Das SR, Emin MT, Wei M, Sun L, Westphalen K, et al. Mitochondrial transfer from bone-marrow-derived stromal cells to pulmonary alveoli protects against acute lung injury. Nat Med (2012) 18:759-65. doi: $10.1038 / \mathrm{nm} .2736$

64. Morrison TJ, Jackson MV, Cunningham EK, Kissenpfennig A, McAuley DF, O Kane CM, et al. Mesenchymal Stromal Cells Modulate Macrophages in Clinically Relevant Lung Injury Models by Extracellular Vesicle Mitochondrial Transfer. Am J Resp Crit Care (2017) 196:1275-86. doi: 10.1164/rccm.201701-01700C

65. Falchi AM, Sogos V, Saba F, Piras M, Congiu T, Piludu M. Astrocytes shed large membrane vesicles that contain mitochondria, lipid droplets and ATP. Histochem Cell Biol (2013) 139:221-31. doi: 10.1007/s00418-012-1045-x

66. Hough KP, Trevor JL, Strenkowski JG, Wang Y, Chacko BK, Tousif S, et al. Exosomal transfer of mitochondria from airway myeloid-derived regulatory cells to T cells. Redox Biol (2018) 18:54-64. doi: 10.1016/j.redox.2018.06.009

67. Puhm F, Afonyushkin T, Resch U, Obermayer G, Rohde M, Penz T, et al. Mitochondria Are a Subset of Extracellular Vesicles Released by Activated Monocytes and Induce Type I IFN and TNF Responses in Endothelial Cells. Circ Res (2019) 125:43-52. doi: 10.1161/CIRCRESAHA.118.314601

68. Boudreau LH, Duchez A, Cloutier N, Soulet D, Martin N, Bollinger J, et al. Platelets release mitochondria serving as substrate for bactericidal group IIAsecreted phospholipase A2 to promote inflammation. Blood (2014) 124:217383. doi: 10.1182/blood-2014-05-573543

69. Wen C, Seeger RC, Fabbri M, Wang L, Wayne AS, Jong AY. Biological roles and potential applications of immune cell-derived extracellular vesicles. J Extracell Vesicles (2017) 6:1400370. doi: 10.1080/20013078.2017.1400370

70. Sandoval H, Kodali S, Wang J. Regulation of B cell fate, survival, and function by mitochondria and autophagy. Mitochondrion (2018) 41:58-65. doi: 10.1016/j.mito.2017.11.005 
71. Liu Y, Lou G, Li A, Zhang T, Qi J, Ye D, et al. AMSC-derived exosomes alleviate lipopolysaccharide/d-galactosamine-induced acute liver failure by miR-17mediated reduction of TXNIP/NLRP3 inflammasome activation in macrophages. Ebiomedicine (2018) 36:140-50. doi: 10.1016/j.ebiom.2018.08.054

72. Lin MT, Beal MF. Mitochondrial dysfunction and oxidative stress in neurodegenerative diseases. Nature (2006) 443:787-95. doi: 10.1038/nature05292

73. Prakash YS, Pabelick CM, Sieck GC. Mitochondrial Dysfunction in Airway Disease. Chest (2017) 152:618-26. doi: 10.1016/j.chest.2017.03.020

74. Park J, Davis RL, Sue CM. Mitochondrial Dysfunction in Parkinson's Disease: New Mechanistic Insights and Therapeutic Perspectives. Curr Neurol Neurosci (2018) 18(5):21. doi: 10.1007/s11910-018-0829-3
Conflict of Interest: The authors declare that the research was conducted in the absence of any commercial or financial relationships that could be construed as a potential conflict of interest.

Copyright $\odot 2021$ She, Xie, Hun, Abdirahman, Li, Wu, Luo, Wan, Wen and Tian. This is an open-access article distributed under the terms of the Creative Commons Attribution License (CC BY). The use, distribution or reproduction in other forums is permitted, provided the original author(s) and the copyright owner(s) are credited and that the original publication in this journal is cited, in accordance with accepted academic practice. No use, distribution or reproduction is permitted which does not comply with these terms. 